\title{
Comment on "Body mass index and contralateral ratio predict outcome following unilateral adrenalectomy in primary aldosteronism"
}

\author{
Jie Weng ${ }^{1} \cdot \mathrm{He} \mathrm{Wu}^{1} \cdot$ Zhiyi Wang $^{1,2}$
}

Received: 7 November 2017 / Accepted: 12 November 2017 / Published online: 12 June 2018

(c) The Japanese Society of Hypertension 2018

We were interested to read the article "Body mass index and contralateral ratio predict outcome following unilateral adrenalectomy in primary aldosteronism", authored by Bokuda $\mathrm{K}$ and colleagues and published recently in Hypertension Research [1]. The authors sought to investigate the preoperative factors contributing to postoperative blood pressure (BP) outcome in patients with primary aldosteronism who had undergone unilateral adrenalectomy. This study was interesting; however, some methodologic issues should be noted.

First, all continuous variables in the study were expressed as the mean \pm s.d. and analyzed by Student's $t$-test. It is widely recognized that the mean \pm s.d. is suitable only for data that follow the normal (Gaussian) distribution [2]. However, the authors ignored the normality of continuous variables in this study (some continuous variables listed in Table 1 clearly did not follow the normal distribution). Medians and interquartile ranges may be more appropriate than means and standard deviations when continuous variables are not normally distributed. We note that the normality assumption can be checked by graphical and statistical methods such as the Kolmogorov-Smirnov test. Nonparametric tests should be used when the normality assumption does not hold. The normality assumption should be considered seriously, as it is impossible to draw accurate and reliable conclusions when the assumption does not hold [2].

\section{Zhiyi Wang}

wzy1063@126.com

1 Department of Emergency Medicine, The Second Affiliated Hospital and Yuying Children's Hospital of Wenzhou Medical University, Wenzhou 325000, China

2 Department of General Practice, The Second Affiliated Hospital and Yuying Children's Hospital of Wenzhou Medical University, Wenzhou 325000, China
Second, the authors constructed a multivariate logistic regression model including all preoperative variables that demonstrated statistical significance $(P$-value $<0.05)$ between the "cured" and "not cured" groups for the BP outcome following unilateral adrenalectomy. First, bivariate correlations among preoperative variables should be assessed to identify potential multicollinearity before multivariate logistic regression analysis; second, a multivariate logistic regression model should be constructed using backward stepwise selection for those variables found to be associated with factors in univariate analysis with $P$-values less than 0.2. In the study conducted by Bokuda $\mathrm{K}$ and colleagues, bivariate correlation between preoperative variables was not assessed, and the preoperative variables with $P$-values less than 0.05 were entered into the multivariate analyses by the author, which can introduce estimation bias into the results [3, 4].

Finally, postoperative systolic BP and postoperative diastolic BP fluctuate within individuals over time, and we suggest that the mean of three or more measurements should have been used in this study. Instead, the authors used only one BP measurement per subject; overlooking within-person variability in this manner can lead to regression dilution bias in the findings [5].

\section{Compliance with ethical standards}

Conflict of interest The authors declare that they have no conflict of interest.

\section{References}

1. Bokuda K, Yatabe M, Mizuguchi Y, Niiyama M, Seki Y, Watanabe D, Yatabe J, Ando T, Morimoto S, Ichihara A. Body mass index and contralateral ratio predict outcome following unilateral adrenalectomy in primary aldosteronism. Hypertens Res. 2017;40:988-93. 
Table 1 Patient characteristics by cured and not cured

\begin{tabular}{|c|c|c|c|c|}
\hline & $\begin{array}{l}\text { All } \\
(n=96)\end{array}$ & $\begin{array}{l}\text { Cured } \\
(n=46)\end{array}$ & $\begin{array}{l}\text { Not cured } \\
(n=50)\end{array}$ & $\begin{array}{l}P \text {-value (cured vs. } \\
\text { not cured) }\end{array}$ \\
\hline Age at surgery, years & $51 \pm 11$ & $47 \pm 12$ & $55 \pm 10$ & 0.0009 \\
\hline Male, $n(\%)$ & $40(41.7)$ & $13(28.3)$ & $27(54.0)$ & 0.0106 \\
\hline BMI, $\mathrm{kg} \mathrm{m}^{-2}$ & $23.3 \pm 3.5$ & $21.8 \pm 3.4$ & $24.6 \pm 3.0$ & $<0.0001$ \\
\hline Complication of DM, $n(\%)$ & $4(4.2)$ & $2(4.4)$ & $2(4.0)$ & 0.9321 \\
\hline Complication of DL, $n(\%)$ & $18(18.8)$ & $5(10.9)$ & $13(26.0)$ & 0.0587 \\
\hline Past history of CVD, $n(\%)$ & $5(5.2)$ & $2(4.4)$ & $3(6.0)$ & 0.7159 \\
\hline Smoking, $n(\%)$ & $32(33.7)$ & $12(26.1)$ & $20(40.8)$ & 0.129 \\
\hline Alcohol, $n(\%)$ & $32(33.7)$ & $15(32.6)$ & $17(34.7)$ & 0.8298 \\
\hline Family history of HTN, $n(\%)$ & $67(69.8)$ & $33(71.7)$ & $34(68.0)$ & 0.6902 \\
\hline Preoperative systolic $\mathrm{BP}, \mathrm{mm} \mathrm{Hg}$ & $140 \pm 17$ & $138 \pm 13$ & $142 \pm 21$ & 0.3419 \\
\hline Preoperative diastolic $\mathrm{BP}, \mathrm{mm} \mathrm{Hg}$ & $85 \pm 12$ & $84 \pm 11$ & $86 \pm 13$ & 0.5771 \\
\hline Number of antihypertensive medications & $1.3 \pm 0.9$ & $1.1 \pm 0.7$ & $1.5 \pm 1.0$ & 0.0338 \\
\hline $\mathrm{K}^{+}$supplementation, $n(\%)$ & $47(49.0)$ & $25(54.4)$ & $22(44.0)$ & 0.311 \\
\hline MRB medication, $n(\%)$ & $65(67.7)$ & $29(63.0)$ & $36(72.0)$ & 0.3485 \\
\hline $\mathrm{K}^{+}, \mathrm{mEq} \mathrm{l}^{-1}$ & $3.2 \pm 0.5$ & $3.2 \pm 0.4$ & $3.2 \pm 0.6$ & 0.5027 \\
\hline $\mathrm{UA}, \mathrm{mg} \mathrm{dl}^{-1}$ & $4.9 \pm 1.4$ & $4.4 \pm 1.1$ & $5.5 \pm 1.4$ & $<0.0001$ \\
\hline 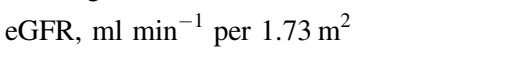 & $\begin{array}{l}78.2 \pm \\
20.8\end{array}$ & $81.7 \pm 16.0$ & $75.0 \pm 24.1$ & 0.1172 \\
\hline PAC, $\mathrm{pg} \mathrm{ml}^{-1}$ & $362 \pm 269$ & $338 \pm 179$ & $384 \pm 332$ & 0.3999 \\
\hline PRA, ng ml ${ }^{-1} \mathrm{~h}^{-1}$ & $0.3 \pm 0.4$ & $0.3 \pm 0.2$ & $0.3 \pm 0.5$ & 0.3729 \\
\hline ARR & $\begin{array}{l}2545 \pm \\
4331\end{array}$ & $\begin{array}{l}2727.3 \pm \\
5522.6\end{array}$ & $\begin{array}{l}2374.7 \pm \\
2843.2\end{array}$ & 0.6939 \\
\hline Urinary aldosterone excretion, $\mu \mathrm{g}$ per day & $\begin{array}{l}24.4 \pm \\
15.2\end{array}$ & $25 \pm 16$ & $23 \pm 14$ & 0.6102 \\
\hline Urinary $\mathrm{Na}^{+} / \mathrm{K}^{+}$excretion & $3.0 \pm 1.5$ & $2.9 \pm 1.6$ & $3.1 \pm 1.3$ & 0.4891 \\
\hline Tumor diameter, mm & $14.6 \pm 5.4$ & $14.3 \pm 4.6$ & $15.0 \pm 6.2$ & 0.5019 \\
\hline \multicolumn{5}{|l|}{ Pathologic diagnosis } \\
\hline Cortical adenoma, $n(\%)$ & $76(79)$ & $37(80.4)$ & $38(79.8)$ & 0.8784 \\
\hline Nodular hyperplasia, $n(\%)$ & $4(4)$ & $0(0)$ & $4(8.3)$ & 0.0454 \\
\hline Cortical adenoma + hyperplasia, $n(\%)$ & $16(17)$ & $9(19.6)$ & $6(12.5)$ & 0.3498 \\
\hline Postoperative systolic BP, $\mathrm{mm} \mathrm{Hg}$ & $125 \pm 12$ & $122 \pm 13$ & $127 \pm 11$ & 0.0375 \\
\hline Postoperative diastolic $\mathrm{BP}, \mathrm{mm} \mathrm{Hg}$ & $79 \pm 8$ & $78 \pm 9$ & $79 \pm 8$ & 0.7381 \\
\hline $\begin{array}{l}\text { Postoperative antihypertensive } \\
\text { medications }\end{array}$ & $0.7 \pm 0.8$ & $0.0 \pm 0.0$ & $1.3 \pm 0.6$ & $<0.0001$ \\
\hline
\end{tabular}

Data are mean \pm s.d. for continuous variables, and number (percent) for categorical variables. Bold values are those found to be significant; $P$-value $<0.05$

$A R R$ aldosterone/renin ratio, $B M I$ body mass index, $B P$ blood pressure, $C V D$ cardiovascular disease, $D M$ diabetes mellitus, $D L$ dyslipidemia, $e G F R$ estimated glomerular filtration ratio, $H T N$ hypertension, $M R B$ mineralocorticoid receptor, $P A C$ plasma aldosterone concentration, $P R A$ plasma renin activity, $U A$ uric acid
2. Öztuna D, Elhan AH, Tüccar E. Investigation of four different normality tests in terms of type 1 error rate and power under different distributions. Turk J Med Sci. 2006;36:171-6.

3. Steyerberg E. Clinical prediction models: a practical approach to development, validation, and updating. Springer Science \& Business Media; New York 2008.
4. Weng J, Wu H, Xu Z, Xi H, Chen C, Chen D, Gong Y, Hua Y, Wang $\mathrm{Z}$. The role of propionic acid at diagnosis predicts mortality in patients with septic shock. J Crit Care. 2017;43:95-101.

5. Masudi S, Yavari P, Mehrabi Y, Khalili D, Azizi F. Regression dilution bias in blood pressure and body mass index in a longitudinal population-based cohort study. Res Health Sci. 2015;15:77-82. 